\title{
Problemas de linguagem na filosofia africana
}

\author{
Language problems in african philosophy
}

\author{
Rogério Saucedo Corrêa
}

Resumo: A comunidade filosófica africana possui um interessante, profícuo e importante debate sobre a linguagem. Por um lado, há os filósofos essencialistas cuja tese principal é o uso das línguas nativas para o ensino e prática filosófica. Por outro lado, há os filósofos não essencialistas que não se opõem ao uso de línguas estrangeiras para o ensino e prática filosófica. Neste artigo apresento os principais pontos deste debate a partir do quadro desenhado por Fayemi e argumento que da tese ideológico-política dos essencialistas não se segue a tese metafísica da impossibilidade de se expressar determinados aspectos da realidade.

Palavras-chave: Linguagem; Filosofia africana; Essencialistas; Não essencialistas

Abstract: The African philosophical community has an interesting, fruitful and important debate on language. On the one hand, there are the essentialist philosophers whose main thesis is the use of native languages for teaching and philosophical practice. On the other hand, there are non-essentialist philosophers who do not opdose the use of foreign languages for teaching and philosophical practice. In this article. I present the main points of this debate from the frame proiected bv Favemi and argue that the political-ideological thesis of the essentialists does not imply the metaphysical thesis of impossibility to express certain aspects of reality.

Keywords: Language; African philosophy; Essentialist; Non-essentialist

\section{Introdução}

Em The problem of language in contemporary African philosophy: some comments ${ }^{1}$, Fayemi oferece um panorama da discussão filosófica africana contemporânea sobre a linguagem. Fundamentalmente, o debate foi suscitado pela obra Philosophy and African Culture $^{2}$, de Wiredu. Nesta ele sustenta que os filósofos africanos devem adotar as línguas nativas para ensinarem aos estudantes temas e questões filosóficas, pois elas são mais apropriadas do que as línguas estrangeiras para se desenvolver esta tarefa. Esta posição faz parte da sua proposta de filosofia da decolonização.

A questão posta por Wiredu desencadeou um debate entre os filósofos africanos, o qual é constituído de duas concepções gerais. Por um lado, os conservadores, e, por outro lado, os progressistas. Os primeiros sustentam que o uso das línguas nativas no filosofar é

\footnotetext{
Professor do Departamento de Filosofia da Universidade Federal de Pernambuco (UFPE). E-mail: rogerio.fsc@gmail.com

${ }^{1}$ FAYEMI, The problem of language in contemporary African philosophy: some comments, 2013.

${ }^{2}$ WIREDU, Philosophy and African Culture, 1980.
} 
fundamental, pois evita distorções e falsas representações dos sistemas africanos de conhecimento e reflexões filosóficas. Nessa linha, além de Wiredu, estão os filósofos Hallene e Sodipo ${ }^{3}$, waThiong'o ${ }^{4}$, Ogunmodede ${ }^{5}$, Gyekye $^{6}$, Bewaji ${ }^{7}$, Uroh $^{8}$ e Afolayan'. Os segundos, é claro, são contra a posição conservadora. Portanto, eles não veem problemas em se usar línguas estrangeiras para fazer filosofia africana. Neste grupo encontram-se filósofos como Bello ${ }^{10}$, Makinde ${ }^{11}$, Tangwa ${ }^{12}$ e Azenabor ${ }^{13}$.

A questão sobre o uso ou não das línguas nativas é importante não apenas para os filósofos africanos, mas para quaisquer filósofos, inclusive os brasileiros, que tenham interesse na filosofia africana. No caso de filósofos negros brasileiros ela é duplamente importante. Suponha que assumo a posição conservadora. Nesse caso, duas consequências podem se seguir. Em primeiro lugar, eu deveria investigar qual a minha ascendência africana para aprender a língua e poder filosofar nesta língua. $\mathrm{Na}$ impossibilidade disso, restar-me-ia escolher arbitrariamente alguma língua africana e aprendê-la. Em segundo lugar, caso não consiga efetuar nenhuma das duas condições anteriores, eu estaria impossibilitado de discutir qualquer problema filosófico compartilhado entre filósofos africanos, pois falo uma língua estrangeira. Pior do que falar uma língua estrangeira, falo uma língua que os filósofos conservadores consideram colonizadora ${ }^{14}$. Evidentemente que uma consequência dessas não é desejável para qualquer um que tenha interesse nas questões e problemas da filosofia africana. No entanto, se os filósofos conservadores estão corretos, as portas para quem não fala uma língua africana, se não estão fechadas, estão semifechadas.

No que segue, reconstruo o panorama apresentado por Fayemi, mas não apenas isso. Apresento uma outra maneira de classificar os dois grupos de filósofos que não como conservadores e progressistas, pois penso que esta classificação não captura um pressuposto filosófico fundamental nesta discussão toda. Dada esta caracterização, formulo um argumento contrário à perspectiva conservadora, o qual não assume o pressuposto que está na base da posição conservadora, a saber, uma concepção essencialista da linguagem.

\footnotetext{
${ }^{3}$ HALLEN; SODIPO, Knowledge, belief, and witchcraft: analytic experiments in African philosophy, 1986.

${ }^{4}$ WA THIONG'O, Decolonizing the mind: the politics of language in African literature, 1986.

${ }^{5}$ OGUNMODEDE, African philosophy in African language, 1993.

${ }^{6}$ GYEKYE, An essay on African philosophical thought: the Akan conceptual scheme, 1995.

${ }^{7}$ BEWAJI, African languages and critical discourse, 2002.

${ }^{8} \mathrm{UROH}$, Colonialism and the language question: a reply to Godfrey Tangwa, 1994.

${ }^{9}$ AFOLAYAN, The language question in African Philosophy, 2006.

${ }^{10}$ BELLO, Philosophy and an African language, 1987.

${ }^{11}$ MAKINDE, African philosophy, culture and traditional medicine, 1988.

12 TANGWA, Colonial legacy and the language situation in Cameroon, 1992.

${ }^{13}$ AZENABOR, Understanding the problems in African philosophy, 2004.

${ }^{14}$ FAYEMI, The problem of language in contemporary African philosophy: some comments, p. 01.
} 


\section{Essencialistas, não essencialistas e consequências}

Um primeiro aspecto importante dessa discussão é a classificação de Fayemi. Segundo esta, o debate contemporâneo dá-se entre conservadores e progressistas. É verdade que ele reconhece outra classificação proposta por Keita ${ }^{15}$ de acordo com a qual se tem fenomenólogos e pragmáticos. Ele não oferece, no entanto, nenhum argumento, explicação ou justificativa quer seja para não adotar a categorização de Keita quer seja para aceitarmos a sua própria classificação. Desse modo, penso que sua proposta tem apenas fins didáticos para lhe permitir discutir seu ponto. De fato, é isso que ele dá a entender quando afirma que sua classificação "é apenas para a conveniência de uma categorização coletiva" ${ }^{16}$. Creio, porém, que categorizar os filósofos como conservadores e progressistas pode induzir a uma leitura menos caridosa. Nesse sentido, os filósofos conservadores seriam menos capazes de reconhecerem as potencialidades da linguagem ou das línguas, ao passo que os progressistas não. O ponto, portanto, é que a leitura pressupõe um juízo de valor sobre os dois grupos. Por essa razão, adoto o termo essencialista para os conservadores e não essencialistas para os progressistas, pois eles são neutros do ponto de vista de juízos de valores e capturam de modo mais adequado um pressuposto filosófico básico desta discussão.

A característica básica da posição dos essencialistas é que existam certos aspectos da realidade que não podem ser expressos ou capturados pelas línguas estrangeiras. Nesse sentido, determinado aspecto da realidade ganense ou nigeriana não pode ser expresso adequadamente no inglês, francês ou alemão. O interessante é que, se isto está correto, nem mesmo o português é adequado para dizer ou capturar este aspecto da realidade, pois ele também é uma língua não nativa. Um exemplo desse tipo de impossibilidade é discutido por Wiredu em Truth and an African Language ${ }^{17}$. Os filósofos não essencialistas discordam desse tipo de abordagem. Uma resposta possível seria dizer que se a língua não é capaz de expressar ou capturar determinado aspecto da realidade, então é necessário aprimorar as ferramentas linguísticas necessárias para tal. Observe que isso é diferente de dizer que é necessário aprimorar a tradução de uma fórmula para a língua Akanda, por exemplo, pois o sentido da via de tradução não é este, mas sim o contrário. É a formalização que deve expressar de modo adequado aquilo que é dito na linguagem ordinária e não o contrário. É verdade que os filósofos precursores da filosofia analítica da linguagem viam a lógica como uma ferramenta de correção da linguagem ordinária. Isso, no entanto, não é uma condição necessária seja para fazer lógica seja para fazer filosofia. Para Russell e Frege era, pois eles viam a linguagem ordinária como um entrave para o

\footnotetext{
${ }^{15}$ KEITA, L, Africa and its linguistic problematic, 1999.

${ }^{16}$ FAYEMI, The problem of language in contemporary African philosophy: some comments, p. 01.

${ }^{17}$ WIREDU, Truth and an African Language, p. 35-50. Mais abaixo, retomo os exemplos de Wiredu.
} 
desenvolvimento do projeto logicista, uma vez que eles consideravam a existência de ambiguidades um entrave para o uso de uma linguagem rigorosa que reduzisse a matemática à lógica. Isso, porém, era um pressuposto dos logicistas. Vejamos, agora, mais pontos do quadro traçado por Fayemi.

O quadro desenhado por Fayemi começa com uma triangulação entre linguagem, pensamento e realidade ${ }^{18}$, pois ele pensa que a filosofia é um estudo da realidade, sua natureza e seus constituintes ${ }^{19}$. Tal tarefa só pode ser levada a cabo por meio do pensamento e da linguagem. A realidade é a totalidade de todas as coisas, estruturas, eventos e fenômenos que atualmente existem, sejam elas observáveis ou não, compreensíveis ou não ${ }^{20}$. O pensamento é a capacidade humana para autoconsciência e conhecimento $^{21}$. Por fim, a linguagem é um fenômeno sócio-cultural usado na comunicação ${ }^{22}$. Após estas breves caracterizações, Fayemi discute a tríade linguagempensamento-realidade no pensamento de Kant, Wittgenstein, Vigotski e Whorf. Tudo isso para mostrar duas coisas. Em primeiro lugar, o fato de que todos estes pensadores concordam que pensamento e linguagem não são idênticos ${ }^{23}$. Nesse sentido, eles são considerados como sustentando uma posição extrema. Em segundo lugar, pode-se pensar que, embora a linguagem não determine necessária e completamente o pensamento, ela afeta os padrões de pensamento das pessoas de uma ou outra maneira. Ela faz isso seja promovendo o significado de alguns contrastes conceituais seja enfatizando outros. Aqui, o ponto visado por Fayemi é fundamental e fica mais claro a partir do argumento que ele oferece. Nesse sentido, dado que pensamentos são flexíveis, não completamente dependentes e simplificados por terminologias linguísticas, segue-se que diferenças na linguagem não significam necessariamente diferenças nos padrões de pensamento. Além disso, dado que a linguagem é generativa e dinâmica, ela não apenas descreve a realidade, mas ela cria a realidade. Como temos diferentes linguagens, então diferentes linguagens criam diferentes realidades. Este argumento contém duas teses. A primeira tese chamo de gap entre linguagem e pensamento. A segunda chamo de tese da criação da realidade. Estas teses são fundamentais para compreendermos o problema da linguagem na filosofia africana, pois, se aceito a tese forte, então a posição dos essencialistas possui um núcleo duro, mas, se aceito a tese moderada, então a posição dos não essencialistas é mais flexível. Vejamos, portanto, o que dizem os essencialistas.

\footnotetext{
${ }^{18}$ Diga-se de passagem, este é um tema clássico da filosofia ocidental. Basta lembrar do Tractatus LogicoPhilosophicus. Ver WITTGENSTEIN, Tractatus Logico-Philosophicus, 1993.

${ }^{19}$ FAYEMI, The problem of language in contemporary African philosophy: some comments, p. 02.

${ }^{20}$ Ibidem.

${ }^{21}$ Ibidem.

${ }^{22}$ Ibidem.

${ }^{23}$ Nesse ponto discordo de Fayemi com relação ao Tractatus Logico-Philosophicus, mas não discutirei isso aqui, pois foge do meu objetivo principal.
} 
Como o principal essencialista é Wiredu, a análise não apenas começa como é centrada nas suas posições. O objetivo principal dele é propor a filosofia da decolonização, a qual é compartilhada pelos demais essencialistas. Aqui, penso que a posição de Wiredu possui duas dimensões que, de certa forma, estão entrelaçadas. Por um lado, ela possui uma dimensão política. Por outro lado, uma dimensão metafísica. A dimensão política configura-se nos atos de resistência ao processo de colonização sofrido pelos povos africanos. A dimensão metafísica diz respeito à perda dos elementos que identificam e individualizam as culturas africanas em função da colonização. Desse modo, o colonialismo caracteriza-se como uma brutal imposição político-cultural por meio de um instrumento fundamental. A imposição de uma educação europeia.

A imposição de uma educação europeia implica na imposição de categorias estrangeiras de pensamento em detrimento dos sistemas africanos de pensamento, com duas consequências importantes. Em primeiro lugar, a produção de distorções das visões africanas de mundo. Isto é a consubstancialização da dimensão metafísica que mencionei acima. Em segundo lugar, a instalação de instabilidades na sociedade africana. Esta, por sua vez, é a consubstancialização da dimensão política. Posto desta forma parece que este processo é ou foi uníssono e totalmente vitorioso. Na verdade, porém, o processo de colonização dos povos africanos não foi algo tão vitorioso assim. Não esqueçamos, inclusive, que boa parte desse processo não se deu por meio de uma aculturação, mas por meio de violência física. De qualquer modo, não creio que a aculturação seja tão eficaz como transparece essa discussão. Aqui, é interessante lembrar o que Appiah diz sobre a África anglófona e francófona cujas populações desenvolveram mecanismos de resistência cultural que lhes permitiram manter suas culturas nativas salvas da colonização ${ }^{24}$. É verdade que corremos um risco de generalizações, pois o foco da discussão é a prática filosófica e a adoção ou não de línguas nativas nestas. No entanto, ela oscila rapidamente e inadvertidamente para dimensões de resistência cultural de um povo, uma vez que a língua de cada povo tem a ver com a sua própria identidade. Deve-se frisar, portanto, que se trata de avaliar a contribuição que a filosofia feita em língua nativa pode ou não proporcionar para a decolonização.

A filosofia da decolonização ou decolonização conceitual é uma reação e resistência à colonização. Para tanto, sua peça principal é o uso das línguas nativas africanas a fim de fazer filosofia. A adoção de línguas nativas africanas é fundamental, pois "o modo como sua linguagem funciona pode predispor você a várias maneiras de falar e, de fato, a várias maneiras de raciocinar" ${ }^{25}$. Não é difícil visualizar a consequência disso para a filosofia. Se sou educado filosoficamente em língua estrangeira, então aprendo a filosofar em uma língua que não é a minha. Logo, raciocino com um esquema conceitual que não é o da minha cultura, pois a linguagem não é neutra. Portanto, a decolonização requer dissociar o

\footnotetext{
${ }^{24}$ APPIAH, Na casa de meu pai: a África na filosofia da cultura, p. 26.

${ }^{25}$ WIREDU apud FAYEMI, The problem of language in contemporary African philosophy: some comments, p. 04.
} 
pensamento filosófico africano das estruturas conceituais impregnadas e transmitidas por meio das línguas estrangeiras. O ápice dessa concepção essencialista é que “... se não usamos línguas africanas na educação, especialmente no pensamento e discurso filosófico, nossa emancipação da dominação colonial permanece incompleta”26. O próximo passo é ver o que os não essencialistas dizem.

Como o principal não essencialista mencionado por Fayemi é Bello, restringirei minha apresentação às suas ideias. Estas se concentram em Philosophy and an African language $^{27}$. Bello tem duas teses centrais ${ }^{28}$. Em primeiro lugar, a recusa de que considerações linguísticas sejam decisivas em disputas filosóficas, uma vez que elas servem apenas para fornecerem dados adicionais. Em segundo lugar, a sugestão de que é necessário cautela por parte dos filósofos essencialistas, pois eles super-enfatizam a importância das suas línguas nativas.

Bello desenvolve seu texto com base na análise de algumas concepções de Wiredu. Nesse sentido, ele reconhece, em débito com o mesmo, ser perfeitamente aceitável que os filósofos africanos dediquem mais atenção e cuidado as suas línguas nativas, uma vez que isso pode gerar não apenas clareza, mas também adicionar dimensões teóricas à filosofia africana. Esse é o caso, em particular, das reflexões de Wiredu em Philosophy and an African Culture $^{29}$. O problema, porém, é que Wiredu mudou de posição em textos posteriores. A mudança, é claro, tem consequências. Tanto em The concept of truth in the Akan language ${ }^{30}$ quanto em The Akan concept of mind $^{31}$, Wiredu abandonou sua posição inicial e adotou uma menos tolerante com as línguas não nativas. Com relação aos conceitos de verdade e fato, por exemplo, ele sustenta que não existe uma palavra equivalente na língua Akan para o sentido cognitivo dos termos "verdade" e "fato"32. Estas noções, porém, podem ser capturadas adequadamente pela frase akaniana "nea eta saa" (what is so ${ }^{33}$ ). Portanto, seja lá o que for dito com o termo "fato" ou com o termo "verdade", pode ser dito usando a frase "nea eta saa". É claro que todas estas teses de Wiredu foram criticadas por outros filósofos africanos. O ponto para Bello, no entanto, não é este, mas sim mostrar as consequências que se seguem delas ${ }^{34}$.

A primeira consequência é a obscuridade na formulação da teoria da verdade como correspondência em língua Akan, pois, quando a frase “ " $p$ ” é verdadeira” significa “" $p$ ”

\footnotetext{
${ }^{26}$ Ibidem, p. 06.

${ }^{27}$ BELLO, Philosophy and an African language, 1987.

${ }^{28}$ Ibidem, p. 05.

${ }^{29}$ WIREDU, Philosophy and African Culture, 1980.

${ }^{30}$ WIREDU, The concept of truth in Akan language, 1985.

${ }^{31}$ WIREDU, The Akan concept of mind, 1983.

32 WIREDU apud BELLO, Philosophy and an African language, p. 05.

${ }^{33} \mathrm{O}$ que é isso.

${ }^{34}$ BELLO, Philosophy and an African language, p. 05.
} 
corresponde ao fato” é vertida para a língua Akan, o resultado é ““ $p$ ' te saa” significa ““ $p$ ' te saa". Isso é assim, dado que os sentidos dos termos "verdade" e "fato" são capturados pela frase "te saa”. A versão inglesa desta frase resulta em “" $p$ ' isso” 35 significa “" $p$ ' isso", que, evidentemente, é uma redundância. A segunda consequência é que existem problemas que podem ser formulados em inglês, mas não podem ser formulados na língua Akan. Por exemplo, a questão “Como proposições verdadeiras relacionam-se com os fatos?” é formulada na língua Akan assim "Como são as coisas que são relacionadas com as coisas que são?”, que, é claro, não faz nenhum sentido. Para reforçar este ponto Wiredu oferece mais um caso exemplar.

Considere as proposições (a) se $p$, então $q$; (b) se apenas $p$, então $q$; (c) $p$ se e somente se $q$; (d) $p$ equivale a $q$; e (e) ' $p$ se e somente se $q$ ' equivale a ' $q$ se e somente se $p$ '. O problema com essas noções lógicas é que apenas os casos (a), (b) e (d) podem ser expressos na língua Akan, mas os demais casos não, uma vez que a língua Akan não tem recursos linguísticos para tal. Se isso é correto, então há noções lógicas que não podem ser expressas na língua Akan. Isso, portanto, reforça a tese de Wiredu segundo a qual existem certos problemas filosóficos que não são universais.

Para Bello, há três pontos fundamentais a serem destacados a partir da análise das posições de Wiredu ${ }^{36}$. Em primeiro lugar, nenhuma linguagem natural é intrinsecamente inferior ou superior a outra. Uma língua pode ser mais desenvolvida que outra língua em algum aspecto específico. No entanto, Bello crê que com uma dose significativa de empenho qualquer língua pode se equiparar a outra. Em segundo lugar, não existe nada de errado em rotular problemas filosóficos como universais, fundamentais ou pseudoproblemas. O problema é se estas classificações podem ser derivadas do fato de que alguns problemas não podem ser expressos numa língua nativa ou sem redundância, como é o caso do problema da verdade. Em terceiro lugar, insights filosóficos podem ser obtidos a partir de fatos linguísticos e a língua de alguém pode ser um index para suas crenças, pressuposições e cultura. No entanto, avalia Bello, devemos ser cautelosos aos usarmos fatos puramente linguísticos para nocautearmos crenças ou doutrinas filosóficas. Pense, por exemplo, no caso das frases "Que sua alma repouse em paz", “Ele perdeu a mente” e "Ele tem uma mente aguçada" ${ }^{37}$. Elas podem dar a entender que quem as profere crê que a alma/mente existe e age independentemente do corpo. Isso, por si só, não implica em um dualismo mente/corpo. Por fim, não é impossível que duas pessoas que falam a mesma língua tenham visões diferentes sobre um mesmo tópico filosófico. Há inúmeros exemplos que se enquadram aqui, mas o de Bello é particularmente interessante. Ele lembra, com

\footnotetext{
${ }^{35} p$ é assim.

${ }^{36}$ BELLO, Philosophy and an African language, p. 06.

${ }^{37}$ Respectivamente "May his soul rest in peace", "He is out of his mind" e "He has a sharp mind". A tradução mais adequada de "He is out of his mind" para o português seria "Ele perdeu a cabeça". No entanto, ela perde o ponto que Bello quer enfatizar.
} 
respeito ao problema da verdade, que Wiredu tende para uma teoria coerentista e pragmática da verdade, uma vez que ele crê que o problema não pode ser expresso em língua Akan. Bedu-Addo ${ }^{38}$, no entanto, discorda de Wiredu e mantém que é possível formular uma versão da teoria da verdade como correspondência em língua Akan.

Para finalizar é interessante apresentar a posição de Bello sobre toda essa discussão. Quanto à possibilidade de se expressar ou não a teoria da verdade como correspondência na língua Akan, ele afirma que se isso não for possível, pior para a língua Akan, pois a inexpressabilidade não diz nada sobre a teoria. $\mathrm{Na}$ realidade, isso diz mais sobre as limitações da língua Akan. Nesse sentido, deve-se observar que a incapacidade da língua Akan expressar a teoria da verdade como correspondência indica que é necessário um maior refinamento ou desenvolvimento dos seus recursos técnicos e linguísticos para que ela possa expressar determinados problemas técnicos localizados.

Embora assuma um lado no debate contemporâneo sobre a linguagem na filosofia africana, Fayemi é um pouco mais moderado que Bello em suas conclusões, pois ele pensa que a divisão entre essencialistas e não essencialistas não é necessária. Pode-se argumentar contra um essencialista, por exemplo, que do fato de se falar e escrever em outra língua que não a sua língua nativa não se segue necessariamente que alguém assuma as ideias e posições colonialistas ${ }^{39}$. De modo análogo, pode-se argumentar contra um não essencialista que é ingênuo supor que do fato de se escrever ou falar em uma língua não nativa segue-se que se escapa das ideias e concepções colonialistas ${ }^{40}$. De qualquer maneira, Fayemi esboça uma agenda para os filósofos africanos ${ }^{41}$. Ao fazê-lo, ele menciona um ponto que considero importante.

A agenda inclui questões que devem ser formuladas, pensadas e respondidas pelos filósofos africanos. Nesse sentido, por que os filósofos africanos continuam fazendo filosofia africana em línguas estrangeiras? Existem esforços sendo realizados para se criar um glossário em línguas africanas ou traduções da filosofia ocidental para as línguas africanas? Dado o grande número de línguas africanas, qual deve ser escolhida para se fazer filosofia africana? Estas questões são importantes; no entanto, mais fundamental, até mesmo mais do que a proposta dos essencialistas, é que se deve entender e superar os desafios de se fazer filosofia africana contemporânea em línguas não nativas ${ }^{42}$. Para tanto, primeiro deve-se perceber a carência de tradição escrita nas línguas africanas. Segundo que as línguas não nativas, em especial as europeias, usadas para fazer filosofia africana são as línguas do poder. Desse modo, se os filósofos africanos compartilham nessas línguas, então eles fazem isso porque estão acostumados a usar estas línguas. Além disso,

\footnotetext{
${ }^{38}$ BEDU-ADDO, J. T, On the Concept of Truth in Akan, 1985.

${ }^{39}$ FAYEMI, The problem of language in contemporary African philosophy: some comments, p. 09.

${ }^{40}$ Ibidem.

${ }^{41}$ Ibidem.

${ }^{42}$ Ibidem.
} 
há uma razão econômica para o uso de línguas não estrangeiras. O uso de línguas nativas implica em uma maior audiência e isso tem implicações práticas, uma vez que um público maior é atingido. Estas razões são muito mais básicas do que promover a comunicação intercultural e a discussão entre os filósofos africanos de diferentes línguas nativas ${ }^{43}$.

\section{Considerações finais}

Neste artigo reconstruí os principais aspectos do quadro traçado por Fayemi do debate contemporâneo da filosofia africana sobre a linguagem. Nesse sentido, por um lado, há os filósofos essencialistas, que propõem o uso das línguas africanas nativas para a prática e ensino da filosofia. Esta posição tem um aspecto político-ideológico e outro metafísico. Por outro lado, há os não essencialistas, que não consideram um problema o uso de línguas estrangeiras para o ensino e prática filosófica.

Nessa discussão creio que há uma distinção importante a ser feita. Ela foi mencionada acima quando falei sobre a agenda proposta por Fayemi. Do meu ponto de vista, ele está correto ao afirmar que as línguas não nativas são as línguas do poder e quanto a isso ele comunga do ideal essencialista. Mas disso não se segue que não se possam usar línguas estrangeiras para fazer filosofia africana. O ponto aqui é delicado. Por isso, necessito fazer uma distinção básica, ou melhor, retomar a distinção entre o nível político-ideológico e o nível metafísico.

Do ponto de vista das ações e procedimentos que uma determinada nação, país ou grupo nacional deve ou não adotar, é plenamente legítimo que os povos africanos implementem o uso das suas respectivas línguas não só para o ensino de filosofia, mas para qualquer atividade. Isso, devo frisar, é uma questão de autonomia político-ideológica ${ }^{44}$. No entanto, ela tem relação com uma questão de natureza metafísica. Não se trata da questão metafísica que a tese essencialista supõe, mas de outra. Se falar uma língua diz respeito à afirmação da identidade de um povo e, de fato, é assim, pois não se diz dos alemães que a língua deles é o francês assim como a língua dos italianos não é o coreano, então falar e usar as línguas nativas africanas é afirmar a identidade de cada um desses povos. No entanto, a tese da afirmação da identidade não se confunde com a tese da existência de aspectos da realidade que não possam ser expressos por outras línguas que aquelas faladas por determinado grupo, como pretende Wiredu. Nesse ponto, meu argumento ecoa o de Bello. Se uma língua, seja lá qual for ela, não tem recursos linguísticos para expressar determinado aspecto da realidade, então isso diz mais da língua e da necessidade de ela desenvolver recursos técnicos para tal do que qualquer outra coisa. Trata-se, portanto, de uma tarefa de refinamento técnico que deve ser levada caso a caso.

\footnotetext{
43 Ibidem.

${ }^{44}$ Para uma análise acurada destas questões, indico o artigo de Pinto. PINTO, J. P. Ideologias linguísticas e a instituição de hierarquias sociais, 2018.
} 


\section{Referências}

AFOLAYAN, A. The language question in African Philosophy. In: OLADIPO. Olusegun. Core issues in african philosophy. Ibadan: Hope Publications, 2006.

APPIAH, A. Na casa de meu pai: a África na filosofia da cultura. Trad. Vera Ribeiro. Rio de Janeiro: Contraponto, 2010.

AZENABOR, G. Understanding the problems in African philosophy. Lagos: First Academic Publishers, 2004.

BEDU-ADDO, J. T. On the Concept of Truth in Akan. In: BODUNRIN, P. O. (ed.). Philosophy in Africa: trends and perspectives. Ife: University of Ife Press, 1985.

BELLO, A. G. A. Philosophy and an African language. Quest. v. 1, n 1, 1987.

BEWAJI. African languages and critical discourse. In: OLADIPO, Olusegun (ed.). The third way in African philosophy: essays in honour of Kwasi Wiredu. Ibadan: Hope Publications, 2002.

FAYEMI, A. K. The problem of language in contemporary African philosophy: some comments. Inkanyiso Journal of Human and Social Science. v. 5, n. 1, 2013.

GYEKYE. An essay on African philosophical thought: the Akan conceptual scheme. Philadelphia: Temple University Press, 1995.

HALLEN, B; SODIPO, J. O. Knowledge, belief, and witchcraft: analytic experiments in African philosophy. Stanford: Stanford University Press, 1986.

KEITA, L. Africa and its linguistic problematic. Quest. v. 13, n 1-2, p. 27-35, 1999.

MAKINDE, M. A. African philosophy, culture and traditional medicine. Ohio: Ohio University Centre for International Studies, 1988.

OGUNMODEDE, F. African philosophy in African language. Proceedings of the international conference on African philosophy and feministic studies. Lagos, 1993.

PINTO, J. P. Ideologias linguísticas e a instituição de hierarquias sociais. Revista da ABPN

- Caderno Temático: letramentos e reexistência. v.10, p. 704-720, 1992.

TANGWA, G. Colonial legacy and the language situation in Cameroon. Quest. v. 6, n 2, 1992.

UROH, C. Colonialism and the language question: a reply to Godfrey Tangwa. Quest. v. 08, n 2, 1994.

WA THIONG'O, N. Decolonizing the mind: the politics of language in African literature. London: James Currey, 1986. 
WIREDU, K. The Akan concept of mind. Ibadan Journal of Humanistic Studies. n 3, p. 113134, 1983.

WIREDU, K. Philosophy and African Culture. Cambridge: Cambridge University Press, 1980. WIREDU, K. The concept of truth in Akan language. In: BODUNRIN, P. O. Philosophy in Africa: trends and perspectives. Ife: University of Ife Press, 1985.

Wittgenstein, L. Tractatus Logico-Philosophicus. Trad. Luiz Henrique Lopes dos Santos. São Paulo: EDUSP, 1993. 